\title{
Alicia Boole Stott's models of sections of polytopes
}

\author{
Irene Polo Blanco
}

Published online: 9 September 2014

(c) Centro P.RI.ST.EM, Università Commerciale Luigi Bocconi 2014

\begin{abstract}
Alicia Boole Stott (1860-1940) was an amateur mathematician who worked on four-dimensional geometry. She is remembered for finding all three dimensional sections of the four- dimensional polytopes (that is, the analogues of the three-dimensional Platonic solids), and for the discovery of many of the semi-regular polytopes. In this text we give a short biography of her and explain her method to calculate the three-dimensional sections of the four-dimensional polytopes. We illustrate her results by showing pictures of her original models and drawings.
\end{abstract}

Keywords Alicia Boole Stott · Polytopes ·

Four-dimensional solids $\cdot$ Higher-dimensional geometry · Ludwig Schläfli · Pieter Hendrik Schoute · H. S. M. Coxeter

\section{Introduction}

The extraordinary amateur mathematician Alicia Boole Stott is remembered above all for her contribution to fourdimensional geometry (Fig. 1). Born in the second half of the nineteenth century, her opportunities for formal education were quite limited. She spent the greater part of her adult life as a full-time wife and mother. In spite of this, however, she achieved important results in mathematics, thanks to her surprising capacity to visualise the fourth dimension. Alicia Boole Stott calculated the three-dimensional sections of regular four-dimensional polytopes (that

I. Polo Blanco $(\bowtie)$

Department Mathematics, Statistics, Computation,

Faculty of Science, University of Cantabria, Avda de los

Castros s/n, 39005 Santander, Spain

e-mail: irene.polo@unican.es is, the analogies to the Platonic Solids in four dimensions) and discovered many of the four-dimensional semi-regular polytopes. In the course of her lifetime, she met two important geometers of the period, P. H. Schoute and H. S. M. Coxeter, with whom she collaborated on various aspects of four-dimensional geometry.

\section{A brief biography}

Alicia Boole Stott, born in 1860 in Castle Road, Ireland, was the third of five children of the famous logician George Boole and his wife, Mary Everest Boole. George Boole died in 1864 at the early age of 49 , when Alicia was only four. His wife was left with the five children, and very little means for maintaining them. For this reason she was forced to move to London with Alicia's four sisters, while Alica was left to live in Cork with another member of the family. At the age of eleven she moved to London to live with her mother and sisters. The four sisters also became important figures of the day for various reasons. For a detailed account of the Boole family we refer the reader to [6]. Figure 2 shows Alicia with her sisters, her mother and several offspring.

It should be noted that the English universities of the time did not offer degrees to women, who could only aspire to study some of the classics of literature and other arts. Alicia's formal scientific knowledge consisted of only the first two books of Euclid. How was it then possible for her to obtain such surprising mathematical results during her life? One of the reasons is undoubtedly due to the unique atmosphere in which she grew up and the special education she received from her mother. Mary Everest Boole was known in her day for her peculiar ideas about education. She wrote several books on mathematics learning, and 


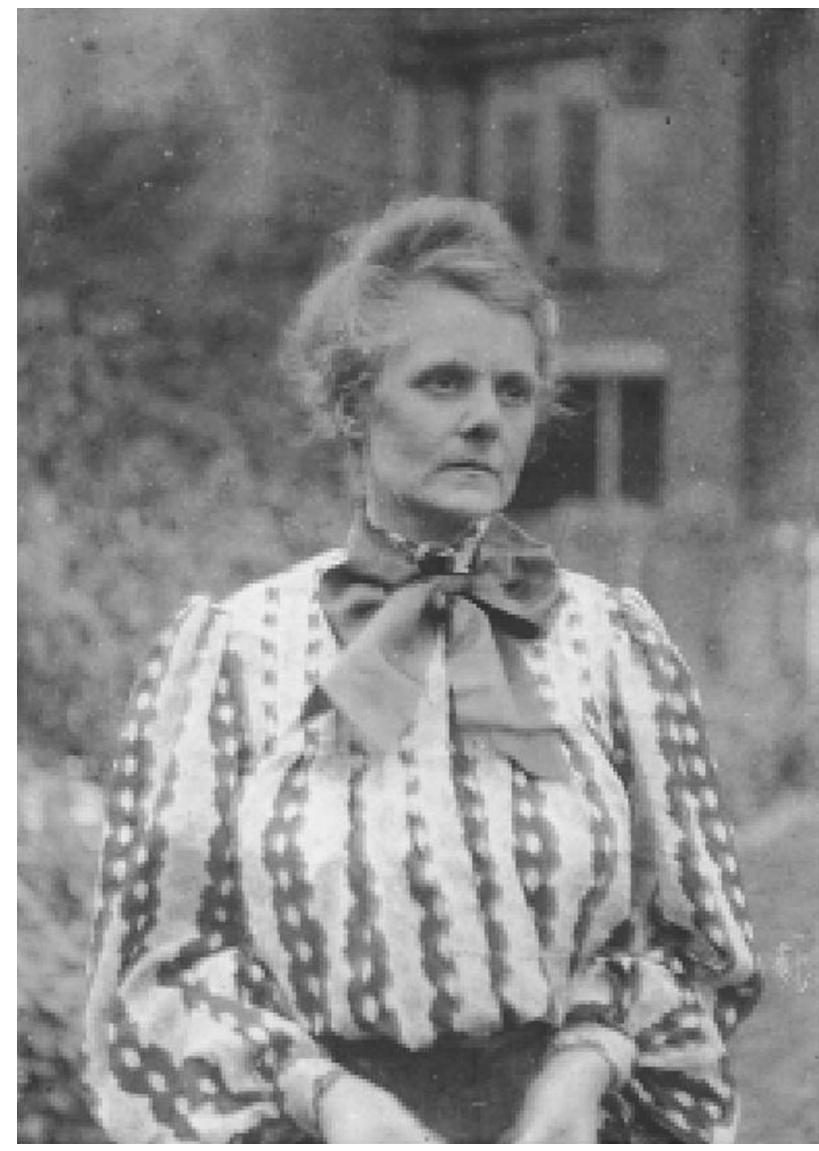

Fig. 1 Alicia Boole Stott (1860-1940). Photo @ University of Bristol Special Collections. Reproduced by permission believed strongly in the importance of early stimulation of children for an effective learning of geometry and other aspects of mathematics. During the years she lived in London, Mary Everest Boole received numerous visitors at home, among whom was the amateur mathematician Howard Hinton. Hinton was a mathematics teacher, and enormously interested in the fourth dimension. He became famous with his book The Fourth Dimension [5], in which the subject is treated from a philosophical point of view. During his visits to the Boole family, Hinton used to pile up groups of wooden blocks to try to allow the five daughters to visualize the four-dimensional hypercube. This greatly inspired Alice in her future work, and she soon began surprise Hinton with her ability to visualize the fourth dimension. Alicia contributed to writing part of the book [4].

In 1890 Alicia married the actuary Walter Stott, with whom she had two children, Mary and Leonard. Inspired by Howard Hinton, Alicia Boole Stott began investigating four-dimensional polytopes in her free time as the children grew. At that time, Boole Stott worked completely independently, without any contact with the scientific world, and proved the existence of the six regular four-dimensional polytopes. These polytopes were first listed by Ludwig Schläfli in 1850 (published after his death in 1901 in [10]), and are four-dimensional analogues of the threedimensional Platonic solids. The six regular four-dimensional polytopes are the hypercube, hypertetrahedron, hyperoctahedron, 24-cell, 120-cell and 600-cell. In addition

Fig. 2 From left to right, top to bottom: Margaret Stott Taylor, Ethel L. Voynich, Alicia Boole Stott, Lucy E. Boole, Mary E. Hinton, Julian Taylor, Mary Stott, Mary Everest Boole, George Hinton, Geoffrey Ingram Taylor, Leonard Stott. Photo (C) University of Bristol Special Collections. Reproduced by permission

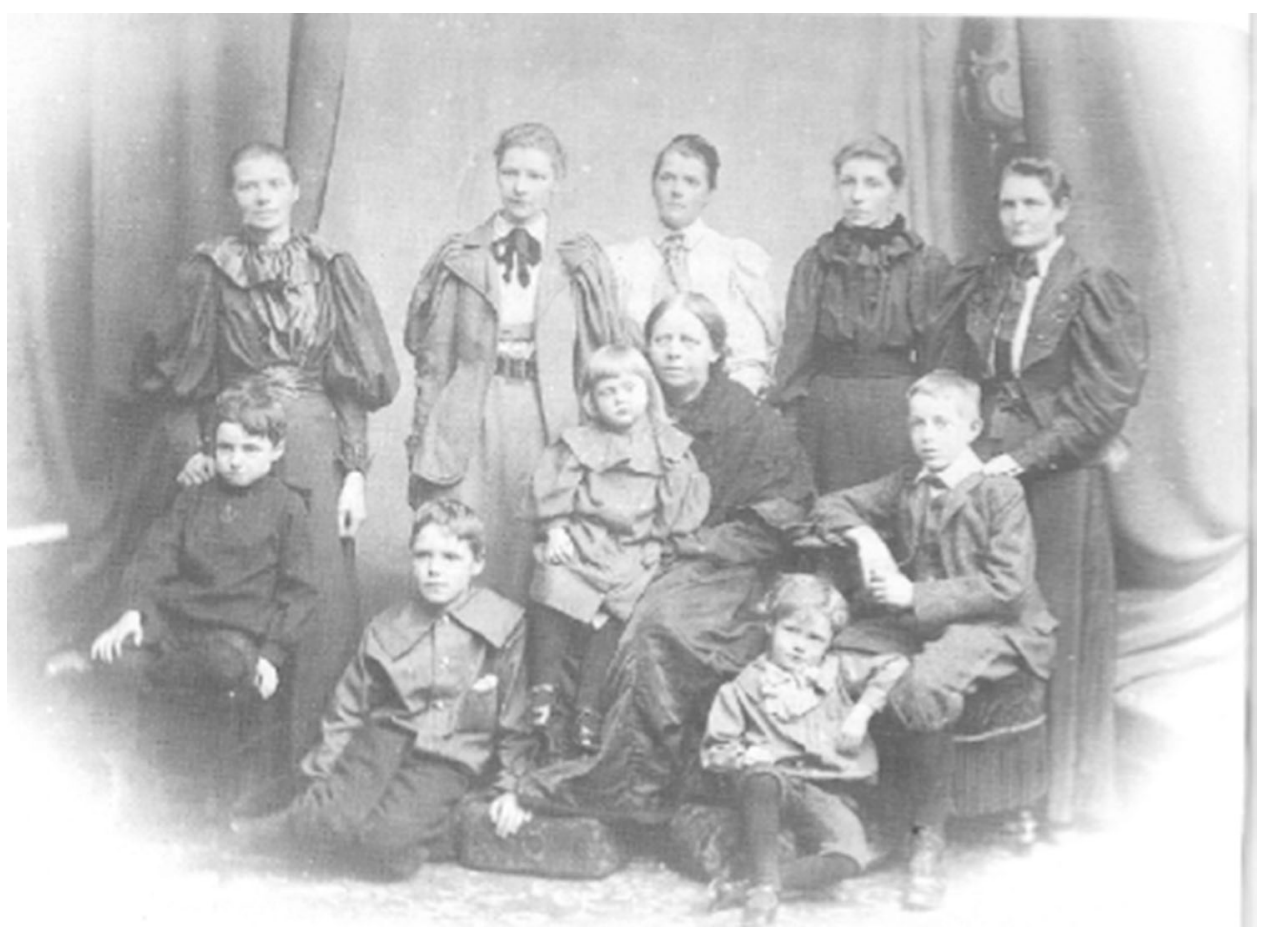



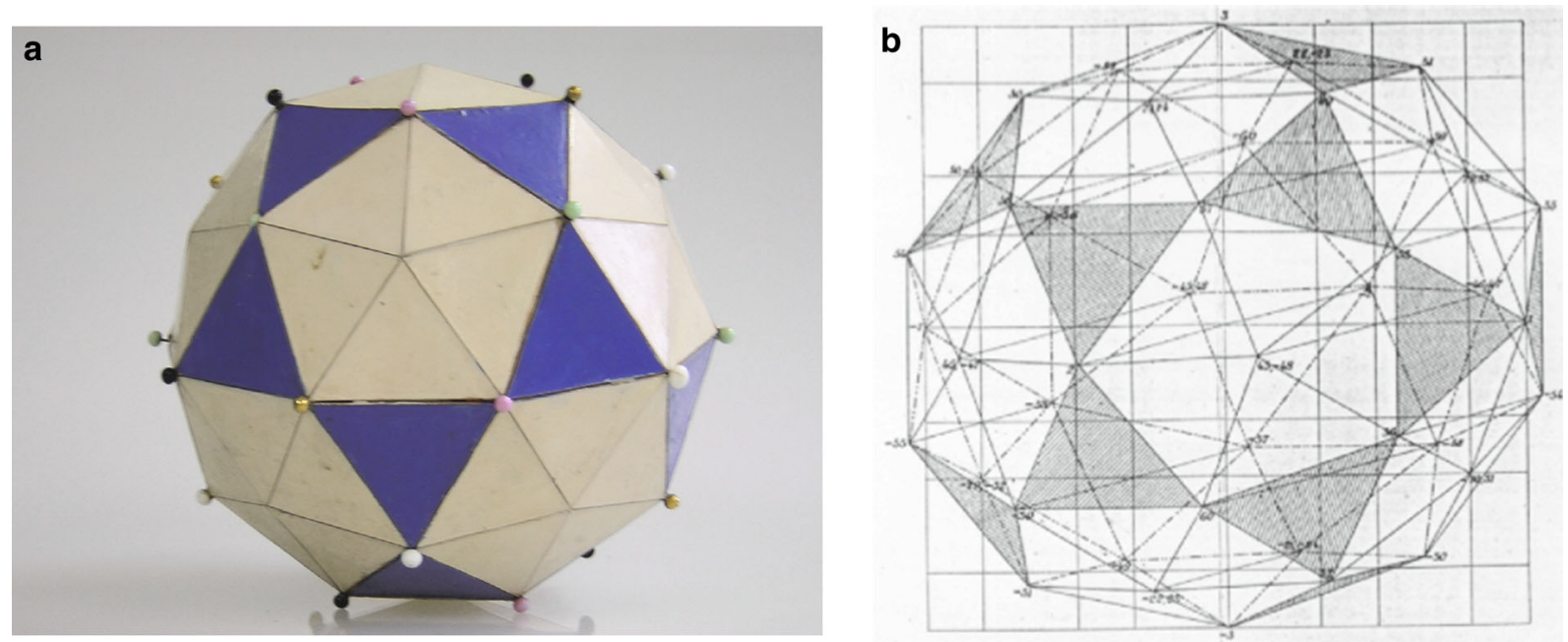

Fig. 3 Central section of the 600-cell. Left the model by Boole Stott; right the drawing by Schoute in [11]

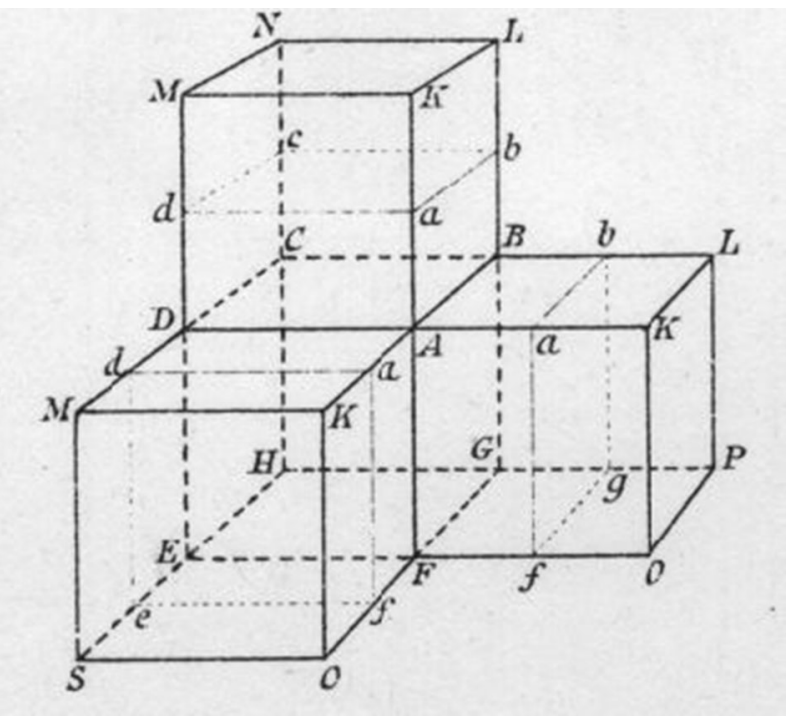

Fig. 4 The three-dimensional unfolding of part of the hypercube (from [1])

to proving the existence of these polytopes, Boole Stott calculated their three-dimensional sections and constructed models of them in coloured cardboard.

In 1894 the Dutch geometer Pieter Hendrik Schoute published an article [11] in which he calculated analytically the central sections of the six regular four-dimensional polytopes. According to Coxeter [3] Boole Stott learned about this publication from her husband. After verifying that Schoute's results coincided with her own, Boole Stott sent pictures of models illustrating not only the central section of each polytope calculated by Schoute, but the entire series. Figure 3 shows that the central sections of Boole Stott and Schoute effectively match for the case of the 600-cell.
Quite surprised by Boole Stott's results, Schoute replied to her immediately, proposing a collaboration that would last for almost 20 years, until Schoute's death in 1913. During this period, Schoute travelled to England during the summer holidays and worked with Boole Stott on various topics regarding the fourth dimension. Their collaboration combined Boole Stott's extraordinary capacity to visualise the fourth dimension with Schoute's analytical method. The work of Boole Stott culminated in an honorary doctorate being awarded to her by the University of Groningen in 1914, in recognition of her contribution to four-dimensional geometry.

After Schoute's death, Boole Stott set her mathematical investigations aside to devote herself exclusively to domestic life. In 1930 she resumed her work when her nephew, the famous physicist and applied mathematician Geoffrey Ingram Taylor, introduced her to the geometer $\mathrm{H}$. S. M. Coxeter. Although Coxeter was only 23 years old and Boole Stott 60, they developed a close friendship and worked together on various aspects of four-dimensional geometry. There are no joint publications, but the contributions of Boole Stott are known thanks to numerous references to them in the work of Coxeter. His book Regular Polytopes [3] also contains numerous facts about Boole Stott's life, and together with [6] is the main source of information about her biography. A more detailed account of Boole Stott's life can also be found in [7].

Boole Stott published her principal mathematical results in two articles: [1] in 1900 and [2] in 1910 (see [9] and [8] for a detailed description of both articles respectively). In what follows we will focus on the first of the publications related to the sections of polytopes and the drawings and models that Boole Stott made of these sections. 

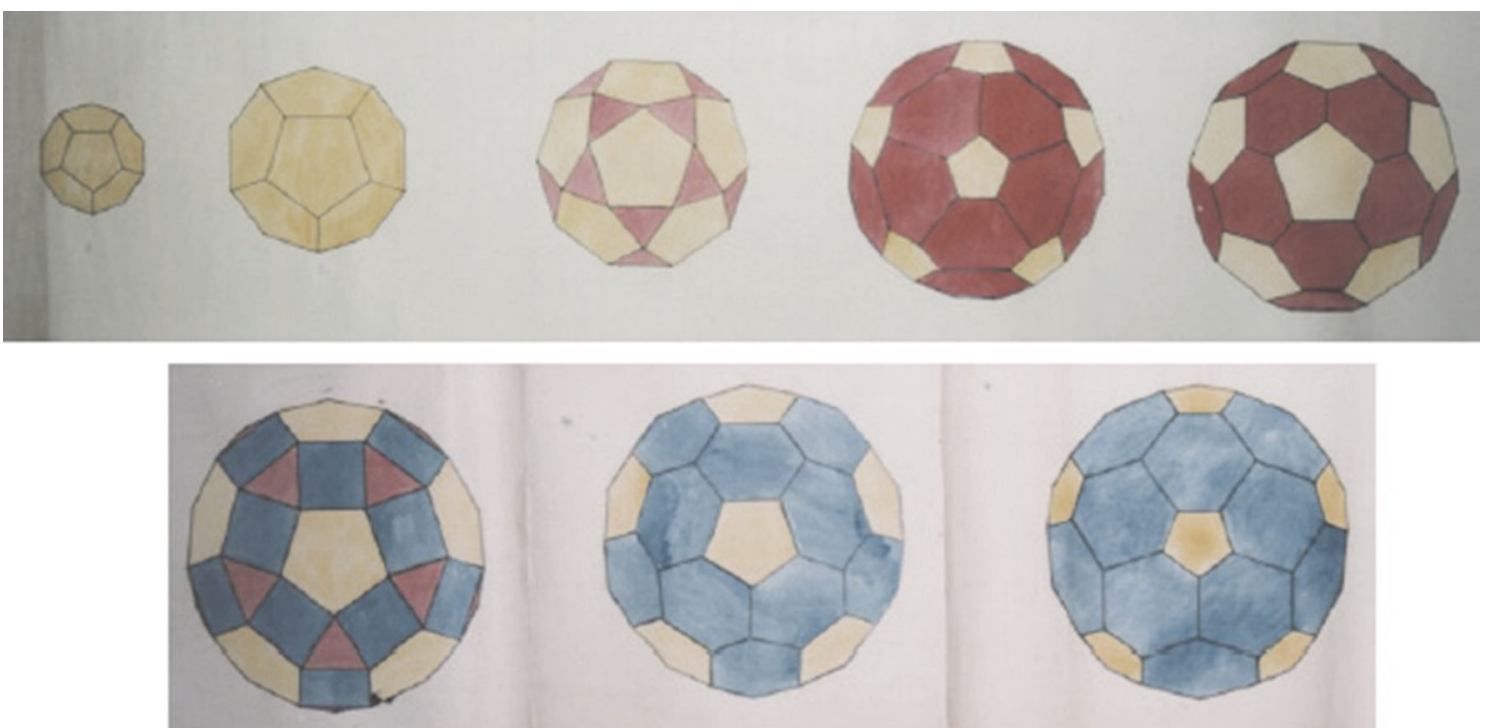

Fig. 5 Drawings of the parallel sections of the 120-cell, conserved at the University of Groningen, The Netherlands. Photo author, reproduced by permission

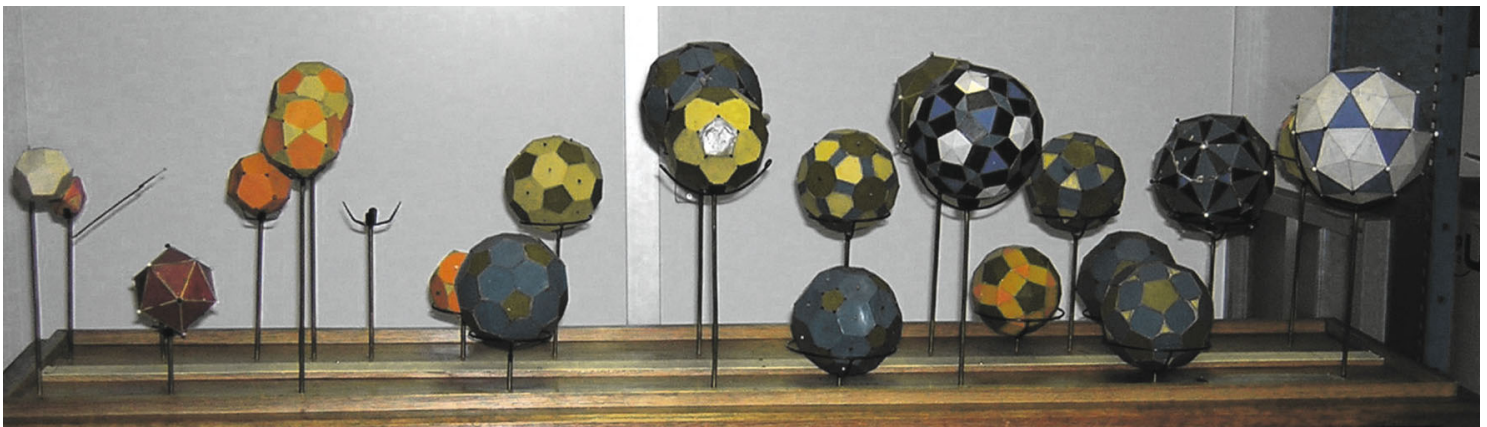

Fig. 6 Models of the perpendicular sections of the 120-cell, conserved at the University of Groningen, The Netherlands. Photo author, reproduced by permission

\section{Three-dimensional sections of four-dimensional polytopes}

Boole Stott's publication of 1900 , 'On certain series of sections of the regular four-dimensional hypersolids' [1] is an exhaustive study of the parallel three-dimensional sections of the six regular polytopes. These sections are the result of intersecting three-dimensional space with the polytope, the three-dimensional space being parallel to one of the three-dimensional faces of the polytope.

With regard to the method used by Boole Stott in her article, several points deserve mention. Just as a Platonic solid can be unfolded in a plane, a four-dimensional polytope can be broken down into three dimensions. Once that is done, the calculations of the sections are considerable simplified, greatly facilitating visualisation.
Figure 4 shows one of her drawings representing part of the unfolded hypercube (note that in the unfolding, several of the vertices, edges, etc. appear more than once, and must be identified in order to reconstruct the original hypercube). A detailed description of this method from a modern point of view can be found in [9].

Boole Stott also studied perpendicular sections of these polytopes, which are characterised by the three-dimensional spaces being considered perpendicular to the segment connecting a vertex with the centre of the polytope. In particular, she made drawings and cardboard models of sections of the two most complex polytopes: the 120-cell and 600-cell. Regarding the sections of the 120-cell (the polytope composed of 120 dodecahedra), we find as many original drawings of the parallel sections as cardboard models of perpendicular sections made by Boole Stott. The drawings in Fig. 5 depict the parallel sections of the 120-cell. 


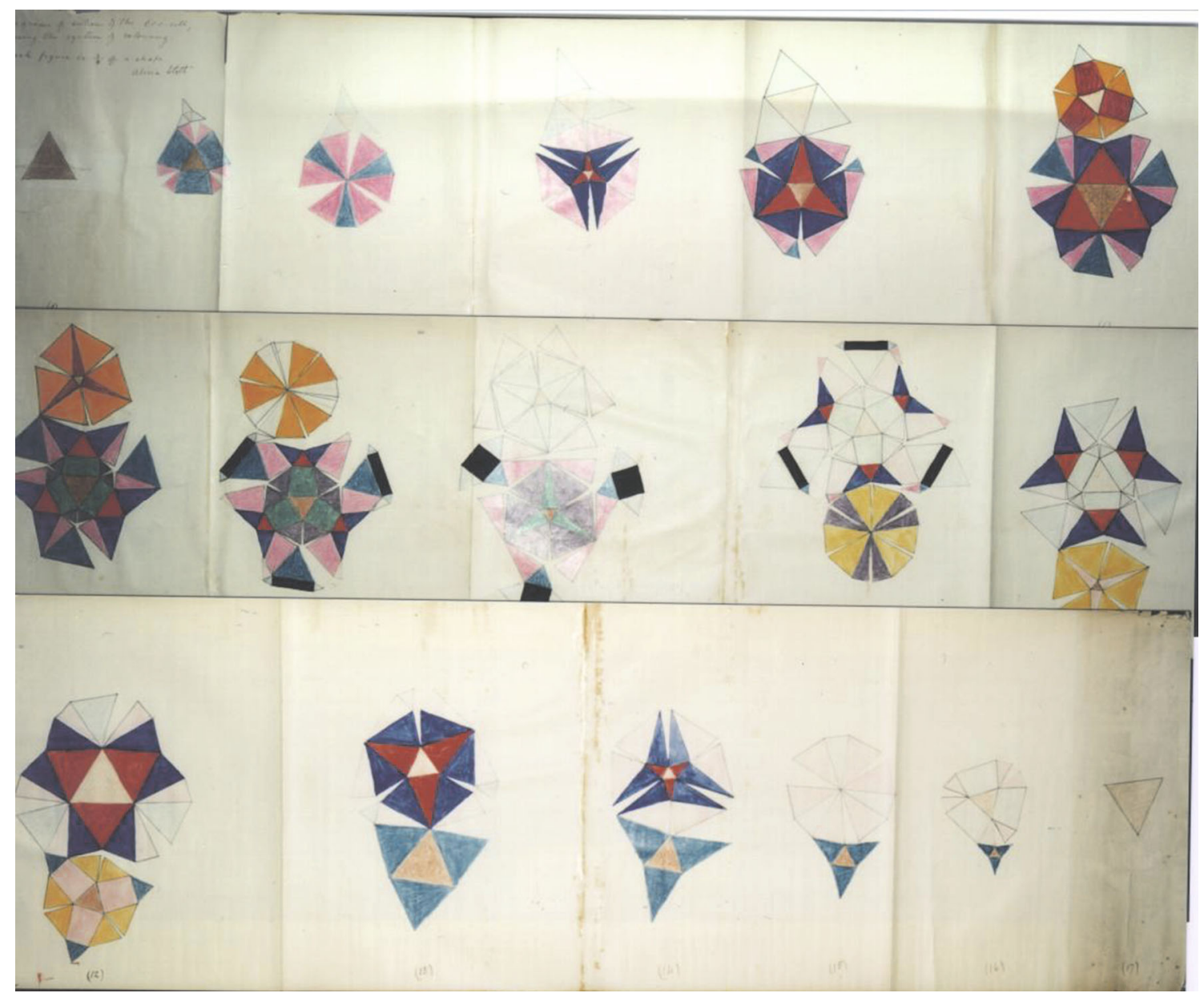

Fig. 7 Drawings of the unfolded planes of the perpendicular sections of the 600-cell, conserved at the University of Groningen, The Netherlands. Photo author, reproduced by permission

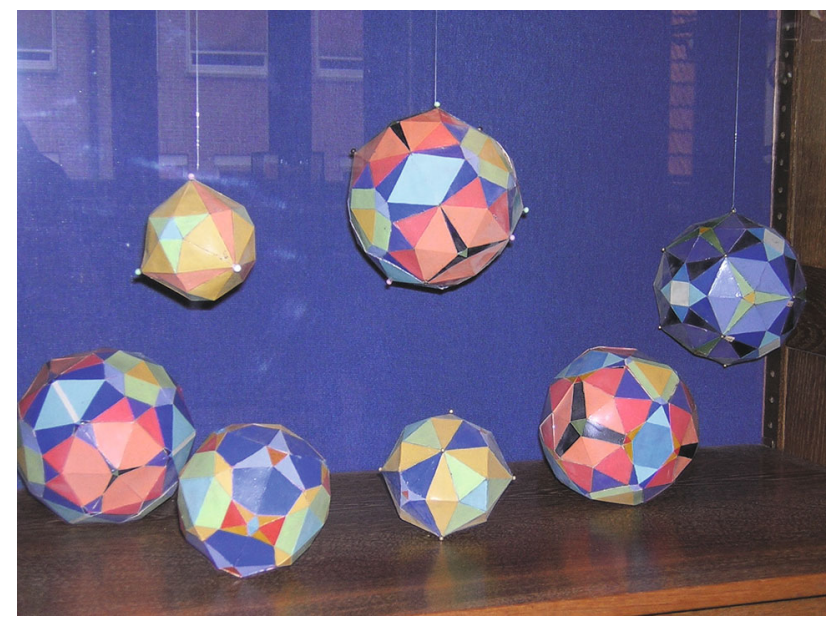

Fig. 8 Models of the perpendicular sections of the 600-cell, conserved at the University of Groningen, The Netherlands. Photo author, reproduced by permission

Furthermore, Boole Stott built models of the perpendicular sections of the polytope. Figure 6 shows these models, today held in the museum of the University of Groningen.
Boole Stott also studied perpendicular sections of the 600-cell and made drawings and cardboard models of these sections. This is the most complex polytope and consists of 600 tetrahedra. At the University of Groningen are found Boole Stott's original drawings corresponding to the unfolded planes of these sections (Fig. 7). We also find models of these perpendicular sections in the museum of the University of Groningen (Fig. 8), and at the University of Cambridge (Fig. 9).

\section{Conclusion}

Alicia Boole Stott is an exceptional example of an amateur mathematician born in the nineteenth century. Isolated from the mathematical community, many of her discoveries were never published. However, this isolation may have helped Boole Stott to develop an intuitive ability to work with the fourth dimension that was very different from the analytical method used at the time, leading her to her discoveries. Boole Stott is remembered today for her 


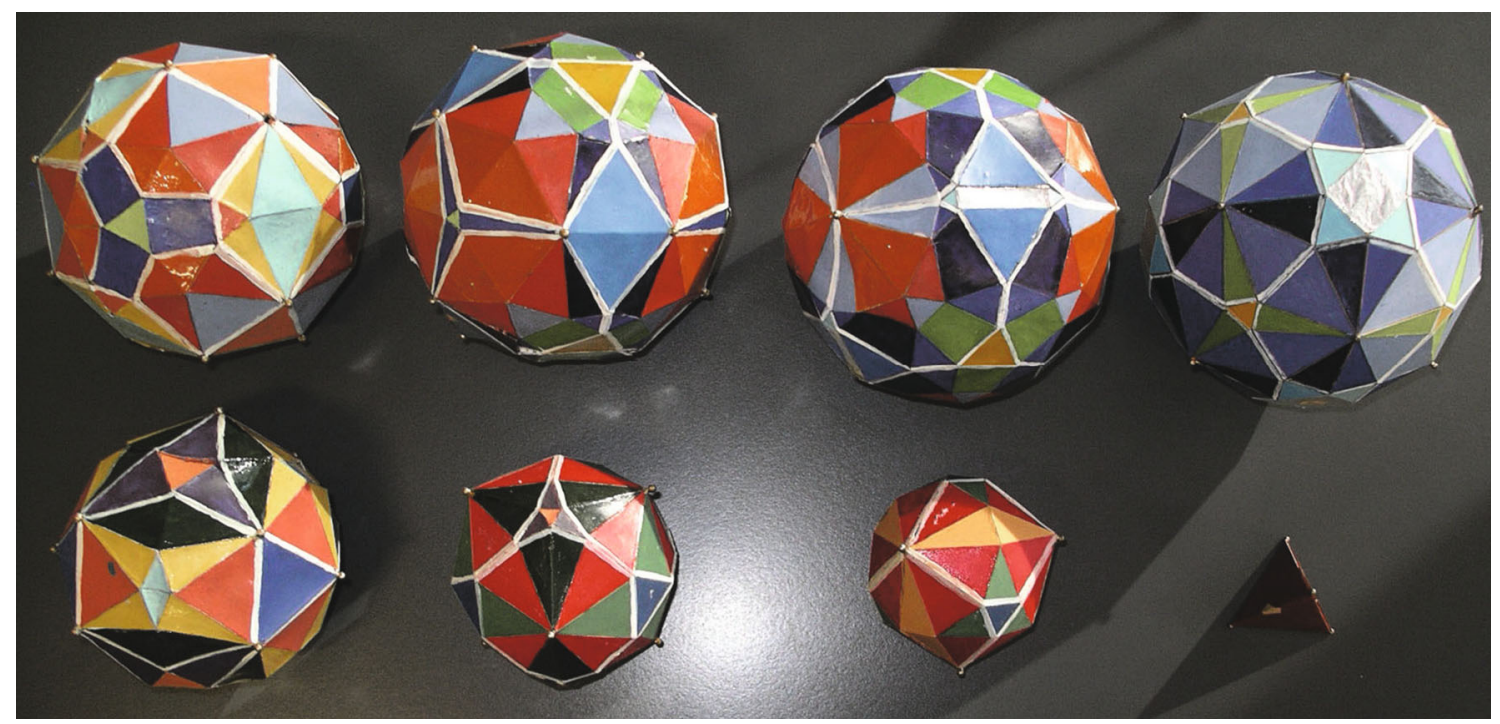

Fig. 9 Models of the perpendicular sections of the 600-cell, conserved at the University of Cambridge, UK. Photo author, reproduced by permission

outstanding contribution to the four-dimensional geometry. Her models and drawings reflect the complexity and beauty of her results.

Translated from the Spanish by Kim Williams.

\section{References}

1. Boole-Stott, A.: On certain series of sections of the regular fourdimensional hypersolids. Verhandelingen der Koninklijke Akademie van Wetenschappen te Amsterdam 7(3), 1-21 (1900)

2. Boole-Stott, A.: Geometrical deduction of semiregular from regular polytopes and space fillings. Verhandelingen der Koninklijke Akademie van Wetenschappen te Amsterdam 11(1), 3-24 (1910)

3. Coxeter, H.S.M.: Regular polytopes. Methuen and Co., London (1948). Rpt. Dover Publications, New York (1973)

4. Hinton, C.H.: A New Era of Thought. Sonnenschein \& Co., Ltd., London (1888)

5. Hinton, C.H.: The Fourth Dimension London. Allen \& Unwin, Crows Nest (1904)

6. McHale, D.: George Boole: His Life and Work. Boole Press, Dublin (1985)

7. Polo Blanco, I.: Alicia Boole Stott, a geometer in higher dimension. Historia Mathematica 35(2), 123-135 (2008)

8. Polo Blanco, I.: A classical approach to the study of Archimedean four-dimensional polytopes. Mathematische Semesterberichte, in press (2014)
9. Polo Blanco, I., Gonzalez Sanchez, J.: Four-dimensional polytopes: Alicia Boole Stott's algorithm. Math Intell 32(3), 1-6 (2010)

10. Schläfli, L.: Theorie der vielfachen Kontinuität. Denkschriften der Schweizerischen naturforschenden Gesellschaft 38, 1-237 (1910)

11. Schoute, P.H.: Regelmässige Schnitte und Projektionen des Achtzelles und des Sechszehnzelles im vierdimensionalen Räume. Verhandelingen der Koninklijke Akademie van Wetenschappen te Amsterdam 2, 3-12 (1894)

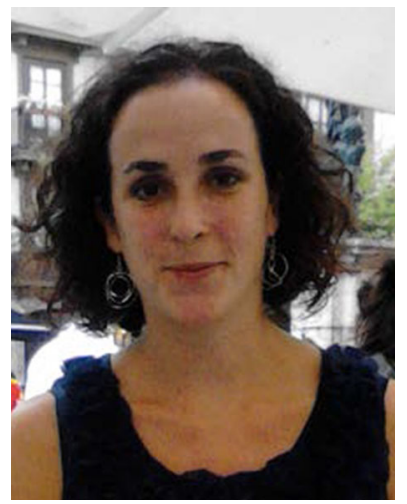

Irene Polo Blanco graduated in mathematics in 2002 from the University of the Basque Country (Spain) and from the University of Groningen (The Netherlands). She received her $\mathrm{Ph} . \mathrm{D}$. from the University of Groningen in 2007. Her thesis concerned the mathematics and the history of nineteenth-century concrete didactic models related to geometry. She currently has a teaching position at the University of Cantabria. Her research interests include algebraic geometry, history of mathematics and didactics of mathematics. 